Artículo de Reflexión

Cómo citar: Fuenmayor, V. y García, E. (2020). Patriarcado, feminismo y construcciones de género en la ficción especulativa The Handmaid's Tale. En: Mediaciones, 25 (16). Pp. 332-346. https://doi.org/10.26620/uniminuto. mediaciones.16.25.2020.332-346

Editorial: Corporación Universitaria Minuto de Dios - UNIMINUTO

Recibido: 11 de febrero de 2020 Aceptado: 23 de junio de 2020 Publicado: 16 de diciembre de 2020

ISSN: 1692-5688 | eISSN: 2590-8057

Verónica Fuenmayor $\mathbf{C}$.

vsfuenmayorcdagmail.com

Magíster Scientiarum en Comunicación Social

Universidad Bolivariana de Venezuela Instituto de Investigaciones de la Comunicación

Universidad Central de Venezuela Venezuela

Erick García Aranguren

erickgarcia1779@gmail.com

Magister Scientiarum en Comunicación Social

Instituto de Investigaciones de la Comunicación

Universidad Central de Venezuela Venezuela

\section{Patriarcado, feminismo y construcciones de género en la ficción especulativa The Handmaid's Tale ${ }^{1}$}

\section{Patriarchy, feminism and gender constructions in speculative fiction The Handmaid's Tale}

\section{Patriarcado, feminismo e construções de gênero na ficção especulativa The Handmaid's Tale}

\section{Resumen}

El presente ensayo pretende dar cuenta de los planteamientos realizados en la serie televisiva "The Handmaid's Tale" (Hulu, 2017) en relación con el feminismo, el sistema patriarcal y su visión con respecto a las construcciones de género. Asumimos dichos planteamientos como un cuestionamiento al sistema patriarcal y a la serie como uno de los ejemplos de ficción televisiva en los que puede evidenciarse la conexión entre el arte y la política, ya que, como plantea Ana María Pérez (2013), "las manifestaciones artísticas son políticas, porque suponen un desacuerdo, una confrontación con las particiones de la realidad sensible. El gran poder de subversión que poseen estas experiencias estéticas en general es su capacidad para ampliar los sujetos, los objetos y los espacios adecuados para el debate, creando nuevos escenarios para la política" (p. 196). En el caso que nos ocupa, consideramos esta ficción

\footnotetext{
1 El presente escrito forma parte de la fase inicial (formulación del proyecto) de la investigación titulada, tentativamente, El Cuerpo: uso y tratamiento en el seriado televisivo distópico, la cual será desarrollada con el auspicio del Instituto de Investigaciones de la Comunicación, ININCO, instituto adscrito a la Facultad de Humanidades y Educación, de la Universidad Central de Venezuela. Dicha investigación iniciará a partir del cuarto trimestre del año 2020.
} 
como una manifestación artística que, además de brindar opciones de entretenimiento, realiza planteamientos que destacan importantes problemas de la cultura occidental y, gracias a su posibilidad de difusión masiva, es un espacio que posibilita la reflexión y el debate en torno a temas políticos de la actualidad.

Palabras clave: Arte, género, patriarcado, política, serie.

\begin{abstract}
The present essay aims to give an account of the approaches made in The Handmaid's tale TV series (Hulu, 2017) in relation to feminism, the patriarchal system and its vision regarding gender constructions. We assume that these approaches are a questioning of the patriarchal system and the series is one of television's fiction examples in which the connection between art and politics is evident, because, as Ana María Pérez (2013) suggests, "the artistic manifestations are political, since they suppose a disagreement, a confrontation with the sensitive reality partitions. The great power of subversion that these aesthetic experiences possess in general is their capability to amplify the subjects, the objects and the adequate spaces for debate, creating new scenarios for politics” (p. 196). In the present case, we consider this fiction an artistic manifestation that, in addition to offering entertainment options, makes approaches that underline important problems of occidental culture and, due to its massive diffusion possibilities, is a space that enables reflection and debate around current political issues.
\end{abstract}

Keywords: Art, gender, patriarchy, politics, series.

\title{
Resumo
}

O presente ensaio pretende dar conta das abordagens realizadas na série televisiva The Handmaid's Tale" (Hulu, 2017) em relação ao feminismo, o sistema patriarcal e sua visão sobre construções de gênero. Assumimos tais abordagens como um questionamento do sistema patriarcal e da série como um dos exemplos de ficção televisiva em que se pode evidenciar a conexão entre a arte e a política, já que, como Ana María Pérez (2013) levanta, "as manifestações artísticas são políticas, porque supõem um desacordo, um confronto com as partições da realidade sensível. O grande poder da subversão que possuem estas experiências estéticas em geral é sua capacidade para ampliar os sujeitos, os objetos e os espaços adequados para o debate, criando novos cenários para a política” (p. 196). No caso em apreço, consideramos esta ficção como uma manifestação artística que, além de oferecer opções de entretenimento, faz abordagens que destacam importantes problemas da cultura ocidental e, graças à sua possibilidade de difusão em massa, é um espaço que possibilita a reflexão e o debate sobre temas políticos da atualidade.

Palavras-chave: Arte, gênero, patriarcado, política, série. 


\section{Series, arte y política}

“Somos mucho más hábiles para fabricar distopías, que para buscar utopías.

Porque somos más hábiles para crear el infierno que para inventar el cielo"

Margaret Atwood

En esta época de múltiples pantallas, tenemos una cantidad formidable de opciones para recibir información de todo tipo, desde noticiosa hasta de entretenimiento. Dentro de los formatos que se encuentran a nuestra disposición, están las series de Tv, cuyos contenidos podemos disfrutar en la actualidad, no sólo en diversos dispositivos sino en el momento en que consideremos más conveniente, gracias al streaming. Esto posibilita que sus contenidos tengan un rango mucho más amplio de acción, es decir, que lleguen a un número mayor de consumidores. Por todo ello, su análisis y estudio resultan de gran interés, puesto que suponen espacios que nos permiten reflexionar sobre nuestra propia realidad, lo que somos y lo que podemos llegar a ser, a través del análisis de las visiones de mundo, representaciones, estereotipos (construcciones sociales) que se exponen en las mismas. Aunque las series televisivas muy pocas veces han sido consideradas lo suficientemente serias y profundas para ser estudiadas académicamente, consideramos que el estudio y análisis de éstas, profundiza y amplía el panorama de las investigaciones sociales, debido a que -como mencionamos recientemente- no sólo brindan entretenimiento, sino que presentan narrativas que exponen grandes problemas de la cultura occidental, entre los que podemos encontrar las construcciones de género.

En tal sentido concordamos con Núñez cuando plantea que:

[...] el conglomerado ideológico que subyace a las "series de TV” y su consumo masivo las convierte en un objeto de estudio interesante, ya que la circulación de ideas no es casual y, como bien sabemos, influye en los espectadores y contribuye en la diseminación y perpetuación de axiologías. En esta lógica, las construcciones de género parecen ocupar un lugar privilegiado en las ficciones más contemporáneas (2017, p. 87).

Agregaríamos nosotros que, además de influir en los espectadores diseminando y perpetuando construcciones de todo tipo, también pueden servir para reflexionar en torno a dichas construcciones de género e incluso cuestionarlas.

En tal sentido y antes de continuar, debemos precisar que consideramos que las series de Tv pueden ser entendidas como piezas de arte que, al presentar planteamientos políticos que muestran grandes problemas de la cultura occidental, pueden contribuir en alguna medida con procesos de transformación social. Tal y como señala Shirley Longan el arte es una filosofía visual y cada vez que un artista logra actualizar los sistemas de pensamiento de su propia época, al hacerlo construye una pieza artística (2011). 
Asimismo, estas piezas artísticas se articulan con la política, en palabras de Ana Pérez (2013) “(...) al propiciar procesos de producción de subjetividades que contribuyan a desencadenar micropolíticas de emancipación y su correlato con relación a la transformación de la sociedad” (2013, p. 193).

Por ello, para este estudio, hemos considerado pertinente analizar la serie televisiva The Handmaid's Tale (creada por Bruce Miller, HULU 2017), puesto que la misma, desde un escenario distópico, expone temas muy propios de nuestra sociedad actual, por ejemplo, el fortalecimiento de ideologías extremistas de corte religioso, que tienen como fundamento el sistema patriarcal con todo lo que éste implica.

En relación con esta serie televisiva, es importante señalar que la misma ha sido incluida por Margaret Atwood (la autora de la novela en la que se basa la serie) dentro del subgénero de la ficción especulativa, en el cual, la historia está compuesta por situaciones reales o que pudieran llegar a serlo. Así, manifestó en una entrevista para El Cultural: "tengo predilección por la ficción especulativa (...) que no es ciencia ficción, no hablo de naves espaciales ni dragones. Es algo bien arraigado a la tierra y pertenece a un futuro muy cercano”. (Seoane, 2017).

En este sentido, la autora ha señalado que, aunque nada de lo narrado en la historia ha sucedido en la realidad, existe siempre la posibilidad de que acontecimientos de este tipo lleguen a cobrar forma, no han sucedido aun porque se ha optado por una solución más ética. Entonces, a diferencia de otros géneros -como la ciencia ficción o el fantasy que buscan crear mundos hasta ahora desconocidos, el carácter especulativo de la obra de Atwood supone un contexto ficcional en el que mezcla historia y futuro, con la intensión de que el espectador adquiera algún tipo de lección moral (Núñez, 2017).

Nuevamente en palabras de Atwood:

Si iba a crear un jardín imaginario, quería que los sapos fueran reales. Una de mis reglas era que no incluiría ningún evento en el libro que no hubiera sucedido en lo que James Joyce llamó la "pesadilla" de la historia, ni ninguna tecnología que no estuviera disponible. Sin artilugios imaginarios, sin leyes imaginarias, sin atrocidades imaginarias. Dios está en los detalles, dicen. Y también el diablo (2017, s.p).

Por todo esto, el objetivo de este ensayo es dar cuenta de los planteamientos de la serie en relación con el sistema patriarcal, las construcciones de género y el feminismo, asumiendo dichos planteamientos como un cuestionamiento de dicho sistema patriarcal y a la serie como un ejemplo de ficción televisiva donde, a través de la conjunción entre el arte y la política, se puede propiciar la reflexión y el debate en torno a temas políticos de actualidad.

2 Traducido del inglés por los autores. 


\section{¿La mujer, nace o se hace? En torno al feminismo, el género y el patriarcado}

“No se nace mujer: una llega a serlo"

Simone de Beauvoir

Antes de comenzar este apartado, es importante aclarar, que no existe consenso en cuanto a las definiciones de ninguno de los tres términos planteados en el título de este punto del escrito. Hay variadas posturas y planteamientos que giran en torno a estos tópicos. Sin embargo, existen puntos coincidentes y compartidos por todas las posturas. A esos planteamientos en común nos referiremos a continuación.

El primer concepto que debemos abordar es el de feminismo. Así, de acuerdo con Carmen Castells:

(...) entendemos por feminismo lo relativo a todas aquellas personas y grupos, reflexiones y actuaciones orientadas a acabar con la subordinación, desigualdad, y opresión de las mujeres y lograr, por tanto, su emancipación y la construcción de una sociedad en la que ya no tengan cabida las discriminaciones por razón de sexo y género (Castells, 1996, 10 citado en Facio \& Fries, 2005, p. 263).

Así, aunque la ideología feminista es plural y diversa, tiene un solo objetivo político: transformar la situación de subordinación de las mujeres en todo el mundo. Pero además, existen otros elementos que comparten todas las agrupaciones feministas, entre los que podemos destacar que: a) todos los hombres y mujeres valemos en cuanto seres humanos; b) todas las formas de discriminación y opresión son igualmente indignas; c) no es aceptable la tendencia en el sistema patriarcal de ofrecer unos valores para guiar las interacciones en lo personal o lo privado (espacio que le correspondería a la mujer) y otros para guiar las interacciones en el mundo de lo público de la política y el poder (espacio que le correspondería al hombre) ya que sólo tiene la finalidad de dominio del hombre sobre la mujer; d) el género es una categoría social igual que la raza, la clase y la edad, que atraviesa todas las otras categorías sociales y es atravesada por éstas y es entendida como una relación de poder; e) la subordinación de las mujeres pretende realizarse además a través del control de los cuerpos, con el fin de controlar la capacidad reproductiva y la sexualidad (Facio \& Fries, 2005), lo que incluye la consideración del carácter institucional y obligatorio de la heterosexualidad (Granados, 2016).

Por su parte, el concepto de género alude, en palabras de Marta Lamas al:

(...) conjunto de prácticas, creencias, representaciones y prescripciones sociales que surgen entre los integrantes de un grupo humano en función de una simbolización de la diferencia anatómica entre hombres y mujeres. Por esta clasificación cultural se definen no sólo la división del trabajo, las prácticas rituales y el ejercicio del poder, sino que se atribuyen características específicas a uno y otro sexo en materia de moral, psicología y afectividad. (2000, p. 3-4). 
Por esto, las teorías de género hacen énfasis en el carácter fundamentalmente social de las características, aptitudes y actitudes atribuidas a las personas en razón del sexo, las cuales son asumidas en el sistema patriarcal como derivadas de la biología y por ende naturales e incuestionables.

La perspectiva de género entonces, pone el acento en la realidad que viven las mujeres, al igual que en los procesos culturales de socialización que éstas internalizan y que refuerzan sus mecanismos de subordinación. Por ello, la perspectiva de género no sólo analiza la relación de subordinación de las mujeres con respecto a los hombres, sino también las relaciones entre mujeres y la funcionalidad de sus prácticas con respecto al sistema patriarcal (Facio y Fries, 2005).

El último término que deseamos exponer aquí es el de patriarcado, el cual, como veremos, en la actualidad está íntimamente relacionado con los otros dos conceptos expuestos anteriormente. Para Marta Fontenla:

El patriarcado puede definirse como un sistema de relaciones sociales sexo-políticas basadas en diferentes instituciones públicas y privadas y en la solidaridad interclases e intragénero instaurado por los varones, quienes como grupo social y en forma individual y colectiva, oprimen a las mujeres también en forma individual y colectiva y se apropian de su fuerza productiva y reproductiva, de sus cuerpos y sus productos, ya sea con medios pacíficos o mediante el uso de la violencia (2008, s.p).

Se trata entonces, de un sistema que justifica la dominación de los hombres sobre las mujeres, apoyándose en una supuesta inferioridad biológica de éstas.

Aunque no todos los sistemas patriarcales son iguales, en todos nos podemos encontrar características comunes: en primer lugar, se trata de un sistema histórico y no natural; en segundo lugar, se sustenta en el dominio del hombre sobre la mujer, ejercido éste a través de la violencia sexual, la cual, es institucionalizada y promovida a través de las instituciones de la familia y el Estado; y en tercer lugar, aunque en los sistemas patriarcales existen hombres en situación de opresión, en todo sistema patriarcal, las mujeres de cada uno de esos grupos oprimidos mantienen una relación de subordinación frente al varón (Facio \& Fries, 2005).

\section{La Serie}

\footnotetext{
“Lo mejor nunca es mejor para todos, siempre es peor para alguien"

Fred (The Handmaid's Tale)
}

La serie “The Handmaid's Tale” (basada en el libro homónimo de la escritora canadiense Margaret Atwood) nos narra un futuro distópico no muy lejano, en el cual, luego de una guerra civil, los EEUU ha quedado reducido a 2 estados -con Anchorage como 
capital-y casi todo el resto del territorio -en Chicago todavía están luchando- está sometido por un gobierno totalitario, teocrático y patriarcal (los Hijos de Jacob) que ha llamado a ese nuevo territorio ocupado: la República de Gilead.

Gilead surge en un momento en el que el medio ambiente ha sido severamente destruido y la capacidad de reproducción de los seres humanos ha mermado considerablemente, hechos que los Hijos de Jacob atribuyen al distanciamiento de las personas con las costumbres más conservadoras. Culpan de la destrucción del mundo al progreso y a las libertades que la sociedad ha alcanzado, haciendo referencia a la contaminación de las fábricas y la radiación de las estaciones, al uso de pastillas anticonceptivas, las pastillas del día después, al aborto y al matrimonio igualitario entre las razones fundamentales por las cuales Dios envió como castigo la infertilidad. Por ello, han instaurado un régimen extremista que controla de manera asfixiante las vidas de todos los que habitan el mismo, a excepción de los Comandantes, quienes ejercen el poder de esta nueva República.

En ese nuevo Estado, se considera que los hombres fueron muy permisivos con las mujeres en el pasado y que, al darles demasiadas libertades, éstas se alejaron y prácticamente abandonaron la función para la cual Dios las había creado, es decir, la reproducción. Por ello, en Gilead, el poder vuelve a estar en manos de los hombres, quienes tienen un mejor criterio y pueden enrumbar al mundo de nuevo por el camino del bien. Para poder realizarlo, han ideado un complicado sistema de castas, que gira en torno de las mujeres fértiles, quienes son las que pueden repoblar el planeta, lo cual se logrará a través de la creación de la figura de la Criada, una mujer fértil, que en su vida pasada fue una pecadora y que ahora tiene la posibilidad de redimirse de todos sus pecados, a través de engendrar hijos para los Comandantes y sus esposas infértiles, quienes se encargarán de educar a dichos niños en los valores de los Hijos de Jacob, para que sean hombres y mujeres de bien. El Cuento de la Criada es la historia de una de estas mujeres: June.

\title{
¿Qué nos cuenta la criada?
}

\author{
“No deberían habernos dado uniformes si \\ no querían que formáramos un ejército" \\ June (The Handmaid's Tale)
}

Luego del visionado de las 3 temporadas (un total de 36 capítulos) de The Handmaid's Tale podemos extraer ejemplos puntuales en los que se encuentran presentes mensajes relacionados con los tres conceptos esbozados previamente, es decir, feminismo, género y patriarcado.

En tal sentido, lo primero que podemos observar en la serie es el énfasis en mostrar el sistema patriarcal, a partir de la exageración al máximo de sus características en el sistema totalitario y teocrático de Gilead.

Así, las mujeres están total y absolutamente sometidas y a merced de los hombres. No pueden trabajar, ni estudiar, ni siquiera leer. Las niñas van al colegio a aprender a 
coser y a servir a sus esposos. Deben desempeñar los roles tradicionalmente asignados a las mujeres: esposa, madre, cocinera, cuidadora del hogar, quien hace las compras, etc. Los trabajadores de las tiendas, choferes, médicos, guardias, todos los demás son hombres. Haciendo clara alusión a los planteamientos realizados por el feminismo y la perspectiva de género en relación con la asignación de roles dicotómicos claramente diferenciados basados en el sexo de las personas y que se instauran a través de procesos de socialización que se mantienen y refuerzan a través de la ideología de las instituciones patriarcales, según las cuales, a las mujeres les corresponden los roles relacionados con el ámbito de lo íntimo y privado del hogar y a los hombres el espacio público de la calle.

Los Comandantes de Gilead han diseñado un complicado sistema de castas en el que cada mujer tiene un lugar y una función determinada. A saber, las mujeres que no son fértiles y que han cometido "pecados" (lesbianas, feministas, adúlteras, etc.) son denominadas "no mujeres” y enviadas a trabajar a Las Colonias, campos de trabajos forzados, donde deben manipular material tóxico y trabajar hasta que les llegue la muerte.

Las mujeres que a pesar de no ser fértiles no son consideradas pecadoras y son solteras o viudas son "Marthas" y cumplen las funciones de amas de llave de los Comandantes.

Otras son Tías. Estas mujeres, en general, están de acuerdo con el sistema y son las encargadas de instruir a las criadas y supervisar su comportamiento. Tienen permiso para disciplinar a través de la tortura a quienes no quieran someterse a su cautiverio.

Un tercer grupo de mujeres no fértiles son enviadas a los Jezabels, burdeles clandestinos donde llevan a los diplomáticos que van a hacer algún trato con los Comandantes, e incluso asiste algún que otro funcionario y los demás se hacen "la vista gorda, porque todos somos humanos, después de todo” (1x08). Allí van:

Todas las mujeres que no pudieron asimilarlo. Algunas eran trabajadoras antes. Esa es profesora de sociología, o era. Tenemos abogadas, gerentes, algunas periodistas. Me dijeron que puedes tener lindas conversaciones con algunas, si lo que quieres es hablar. Tenemos una gran colección. Prefieren estar aquí (1x08).

Le dice Fred a June la primera vez que la llevó a uno de estos sitios para poder estar con ella fuera de La Ceremonia.

Las mujeres que estaban casadas antes del golpe de estado y no pertenecen a la élite gobernante, son llamadas "Econoesposas" y pertenecen a la clase de la "Econogente" o "Econopersonas". Pueden permanecer con su familia e hijos mientras sigan las leyes de Gilead, pero si no acatan las normas y se someten, les quitan a sus hijos y las convierten en Criadas.

Por otra parte, encontramos a las Esposas que, aunque tienen una posición social privilegiada en relación con las demás, también están sometidas a las decisiones y los deseos de sus esposos. 
Obviamente están las Criadas: mujeres fértiles, pero pecadoras en su vida anterior, que ahora tienen la oportunidad de sacrificarse por la humanidad para redimir sus pecados. Deben servir en las casas de los Comandantes y realizan "La Ceremonia”, para concebir el hijo de éste con su Esposa, gestarlo, amamantarlo y después entregárselo a ellos y seguir a su próxima casa donde deberán hacer todo de nuevo con su nuevo Comandante. En cada casa adoptarán el nombre del Comandante de turno, así, nuestra protagonista (June) primero es OfFred (DeFred) y después OfLawrence (DeLawrence).

Es importante puntualizar que La Ceremonia consiste en un ritual que se produce una vez al mes (en el momento de la ovulación de la Criada), en el cual ésta es colocada boca arriba sobre la cama matrimonial de su Comandante, con la cabeza entre las piernas de la Esposa y las piernas colgando en el borde de la cama. Las manos de la Criada son sujetadas por la Esposa, quien observa como entra en la habitación el Comandante, totalmente vestido, y sin ver a la Criada, la penetra para fertilizarla.

Este ritual se basa en un pasaje bíblico que narra la historia de Jacob y su esposa Raquel, y dice lo siguiente:

Viendo Raquel que no daba hijos a Jacob, tuvo envidia de su hermana, y decía a Jacob: Dame hijos, o si no, me muero. / Y Jacob se enojó contra Raquel, y dijo: ¿Soy yo acaso Dios, que te impidió el fruto de tu vientre? / Y ella dijo: He aquí mi sierva Bilha; llégate a ella, y dará a luz sobre mis rodillas, y yo también tendré hijos de ella. / Así le dio a Bilha su sierva por mujer; y Jacob se llegó a ella. / Y concibió Bilha, y dio a luz un hijo a Jacob. / Dijo entonces Raquel: Me juzgó Dios, y también oyó mi voz, y me dio un hijo... (1x01)

En relación con "La Ceremonia” hay una voz en off de Emily, otra de las Criadas, quien describe su experiencia del proceso y cómo lo afronta para poder llevarlo a cabo:

Lo tratas como si fuera un trabajo, un trabajo desagradable, que hay que terminar lo antes posible. Esto lo hace soportable. Uno se separa de uno mismo. Uno describe un acto de reproducción, de fertilización tal vez. Para ti es como una abeja para una flor, nada más. Te armas de valor, finges no estar presente, no estar en tu cuerpo. Dejas tu cuerpo (2x10).

Otro ejemplo donde puede observarse la dominación del cuerpo de las mujeres por parte de los hombres en Gilead es cuando a Emily le amputan el clítoris porque descubren que tiene una relación con una de las Marthas. Deciden perdonarle la vida puesto que su útero y sus ovarios están en buen estado y en Gilead lo más importante son los hijos, pero eliminan la posibilidad de que pueda experimentar placer sexual. La Martha con quien mantiene la relación no corre con tanta suerte, ya que, al no ser fértil no es útil a los intereses de esta nueva República, por lo que es condenada a muerte y ahorcada frente a Emily.

Las Criadas son, entonces, en palabras de June: "úteros con patas" (1x02) que no tienen ningún tipo de poder o control sobre sus cuerpos o sus vidas. Estas prácticas 
coinciden con los planteamientos realizados por Faco y Fries en relación con el sistema patriarcal:

Toda forma de dominación se expresa en los cuerpos ya que son estos en última instancia los que nos dan singularidad en el mundo. En el caso de las mujeres, el disciplinamiento ha sido ejercido por los hombres y las instituciones que ellos han creado (la medicina, el derecho, la religión) con el fin de controlar la sexualidad y la capacidad reproductiva de las mujeres, expresión de la diferencia sexual (2005, p. 267).

Hay otros ejemplos relacionados con el control del cuerpo que no tienen conexión directa con la sexualidad, pero que se aplican como señal de dominio y son las mutilaciones corporales que se utilizan como castigo por la transgresión de alguna norma. Así, a Jeannine (una Criada) le sacan un ojo porque se niega a seguir las reglas en el Centro Rojo (lugar de adiestramiento para las Criadas), a OfGlen le cortan la lengua porque se atrevió a hablar y contrariar una orden de Tía Lydia (una de las Tías del Centro) y a Serena, a pesar de ser Esposa de uno de los Comandantes con más poder en Gilead (Fred), le cortan un dedo porque se atrevió a leer un pasaje de la Biblia para apoyar en las escrituras su petición de que también las niñas pudieran aprender a leer para que tuvieran acceso al contenido de la Biblia de primera mano.

Por todo esto, refiriéndose al papel que desempeñan todas las mujeres en Gilead, June dice: "Esposa, Criada, Martha, madre, hija, novia, reina, perra, criminal, pecadora, hereje: prisionera” (2x13).

Podemos ver aquí, todo el cuestionamiento del sistema patriarcal, a través de la exageración de la situación distópica: dominio del hombre sobre la mujer, hasta el extremo de someter su cuerpo, evitar que pueda estudiar e incluso aprender a leer, para aumentar su sometimiento y dependencia y prevenir que tenga alguna posibilidad de rebelarse; énfasis en el papel que debe asumir la mujer, directamente ligado a su sexo; asociación directa entre el sexo y su finalidad en la vida, la maternidad, el cuidado del hogar, del esposo y los hijos.

Lo que nos lleva al segundo punto: los planteamientos de género. En la serie estudiada hay también varios ejemplos representativos que podemos mencionar.

Uno de ellos es cuando Fred -el jefe de la casa donde le corresponde ser Criada a Juneconversa con June y le explica desde su punto de vista las ventajas que las mujeres tienen en Gilead, le dice: "ahora tienen respeto, se las protege, pueden cumplir su destino biológico en paz”, June le pregunta: “¿destino biológico?” Y Fred le responde: “hijos” (1x05), aludiendo también a los roles de género establecidos en el sistema patriarcal.

Otro ejemplo lo encontramos en una de las primeras escenas de la serie donde podemos ver a la Tía Lydia dándoles una lección a las Criadas a su cargo sobre cómo 
deben comportarse de ahora en adelante y cuál va a ser su función. Así les dice que: (...) "tienen el regalo de la fertilidad, que es un regalo de Dios. Dios las dejó intactas para servir al propósito bíblico como Bilha sirvió a Raquel. Son privilegiadas porque le van a engendrar hijos a los Comandantes” (1x01).

Más adelante en la misma escena les dice: "Sé que todo esto les debe parecer muy raro. Pero "lo normal" no es más que aquello a lo que nos acostumbramos. Puede que esto no les parezca normal ahora mismo... pero acabará siéndolo. Esto se convertirá en los normal” (1x01). Podemos ver en este ejemplo, como se alude al carácter social de los roles de género.

En relación con la orientación sexual, las personas homosexuales son consideradas en Gilead “Traidores del género”. Emily es lesbiana -como ya mencionamos- y en la vida de antes estaba casada con otra mujer y tenían un hijo, por eso para la Tía Lydia Emily es: "una ofensa de Dios, una bestia repulsiva" (1x03). Podemos observar en este punto como esta postura concuerda con lo expresado anteriormente en relación con que parte de la dominación de los cuerpos en el sistema patriarcal, pasa por la institucionalización y la obligatoriedad de la heterosexualidad. En este aspecto, Marta Lamas plantea que:

El género produce un imaginario social con una eficacia simbólica contundente y, al dar lugar a concepciones sociales y culturales sobre la masculinidad y la feminidad, es usado para justificar la discriminación por sexo (sexismo) y por prácticas sexuales (homofobia) (2000: 4).

Por último, en la serie también se hace referencia al feminismo, al activismo y a la unión de las mujeres como modo de lucha contra la situación de opresión en la que se encuentran, aludiendo sin nombrarlo, al concepto de sororidad.

Así, al final de la primera temporada, las Criadas son llamadas a una actividad que denominan "salvamento", en la cual éstas son conminadas a apedrear, patear, ahorcar, a alguna persona que haya cometido un delito y haya sido condenada a muerte por ello. Estando ya en el sitio donde deben apedrear -esta vez- a la persona, se percatan de que a quien tienen que apedrear hasta la muerte es a Jeannine, una Criada que se ha convertido en compañera y amiga de las demás. Ante esta realidad, ellas se niegan a hacerlo, con lo cual, realizan su primer acto de rebeldía conjunta.

Otro ejemplo podemos encontrarlo cuando June logra sacar de Gilead a su hija Nichole, gracias a una red de ayuda formada por Marthas y Criadas. Luego de que le entrega a Nichole a Emily, para que escape con ella a Canadá, dice: "la victoria de una es la victoria de todas" (3x05). En otro momento, en el que se siente un poco más segura, pues empieza a ver que las Criadas comienzan a unirse, se escucha lo siguiente en voz en off:

En el Centro Rojo vi una mirada que muy poco se veía en la vida real, esa mirada era terror. Ya no nos miramos de ese modo. Es culpa suya. No debieron habernos dado uniformes si no querían que formáramos un ejército (1x10). 

de dice:

Otra frase de June que se refiere a la unión entre las mujeres es esta voz en off don-

Hubo un tiempo en que las mujeres podían elegir. Nuestra sociedad se movía, como diría Tía Lydia, por la cantidad de opciones. "Conocemos los sacrificios que deberán realizar" diría ella. "Es lamentable cuando los hombres las denigran”. (...) Madre, donde sea que estés, ¿puedes oírme? Querías una cultura propia de las mujeres, pues, ahora hay una. No es lo que pensabas, pero existe. Y esto es lo que hacemos: los observamos, a los hombres. Los estudiamos, los alimentamos, los complacemos, podemos hacerlos sentir fuertes o débiles. Los conocemos así de bien. Conocemos sus peores miedos. Y con algo de práctica, en eso nos convertiremos: en sus peores pesadillas. Un día, cuando estemos listas, iremos por ustedes. Sólo espérennos (3x03).

Y un último ejemplo es cuando Moira (mejor amiga de June antes de Gilead y que fue Criada y luego prostituta en Jezabels antes de escapar a Canadá) habla con Serena (quien es Esposa de un Comandante muy poderoso y co-creadora de Gilead) y le dice:

Que te vistas con otra ropa no cambia quien eres. Sigues siendo la misma mujer que sostuvo a mi amiga para que tu marido la violara [haciendo alusión a "La Ceremonia”]. (...) También me violó a mí en el prostíbulo. Me trató como una mierda, como si no valiera nada. Mira, soy quien soy. He pecado mucho, pero tú... Tú eres la traidora de género (3x12).

Haciendo alusión a que a Moira también la llamaban de ese modo en Gilead por ser lesbiana y al comportamiento insolidario y egoísta de Serena para con las demás mujeres en Gilead, el cual, como mencionamos anteriormente, es funcional al sistema patriarcal, mucho más en el caso de este personaje, puesto que Serena fue promotora del golpe de estado y creadora de muchos de los preceptos en los que se basó luego Gilead.

Toda la serie tiene un planteamiento feminista al querer liberar a las mujeres de la opresión masculina. Podemos resumir esta postura planteada en la serie en estas palabras de Granados:

Un primer elemento a destacar es el compromiso básico del movimiento teórico y político feminista en dar fin al patriarcado, de tal forma que se subvierta una supremacía masculina considerada referente para el desarrollo de todos los ámbitos de la vida social y por lo tanto subordinadora de valores femeninos expresados en el mundo material y simbólico. Pese a la diversidad de posturas generadas en diversos momentos históricos y culturales del movimiento, la identificación de este sistema de opresión es clave y parte del trabajo académico y militante pone de relieve la necesidad de ubicarlo y contextualizarlo permanentemente para avanzar en el reto cultural de entenderlo tanto como las exclusiones, discriminaciones y violencias que genera (2016, p. 103). 


\section{A modo de conclusión}

Luego de todo lo planteado, podemos evidenciar -primeramente- que se puede observar la relación entre el arte y la política cuando nos encontramos con una serie como "The Handmaid's Tale", que cuenta de una manera tan comprometida una situación que, aunque es ficcional, pudiera llegar a convertirse en realidad si no prestamos suficiente atención a los cambios, tal vez sutiles que se vienen produciendo a nivel político y social en el mundo actual. Poner el acento en estos aspectos puede servir para tratar de impulsar el cambio social. Esto lo logra la serie a través de la exposición de situaciones que muestran de una manera muy clara y cruda el sistema patriarcal llevado al extremo. Como lo plantea Lorenzo Agüero:

Las distopías son representaciones artísticas de sociedades totalmente indeseables, pero por sobre todo, son una señal de alerta. Las distopías nos alertan sobre lo que pasaría si nos descuidamos y no revertimos los aspectos más preocupantes de nuestro tiempo. En el siglo pasado, marcado por los totalitarismos y el consumismo, fueron 1984 (George Orwell) y Un mundo feliz (Aldous Huxley) las obras que nos hicieron abrir los ojos sobre los peligros que el siglo XX traía consigo (2018, s.p).

En este sentido, el mensaje más importante que, a nuestro criterio, trasmite la serie es que debemos estar atentos a los cambios, por muy sutiles que sean, porque si no lo hacemos, tal vez cuando nos demos cuenta sea demasiado tarde. June lo expresa muy bien cuando dice:

Ahora estoy despierta y veo el mundo. Antes estaba dormida. Así es como permitimos que sucediera. Cuando masacraron el Congreso no despertamos. Cuando culparon a los terroristas y anularon la Constitución tampoco despertamos. Dijeron que sería temporal. Nada cambia de golpe. Si estuvieras en una bañera que se calienta poco a poco morirías hervida antes de darte cuenta (1x03).

Adicionalmente, es necesario resaltar la importancia que tienen los medios de comunicación masiva, y los productos que en ellos se transmiten, puesto que posibilitan que sus contenidos sean consumidos por una cantidad muy grande de personas, con lo cual la posibilidad de que las ideas y valores que se transmiten en ellos se diseminen, se incrementa grandemente, en comparación con otros medios que no tienen tanto alcance.

Por último, debemos hacer énfasis en el potencial que tiene las series de televisión, como obras de filosofía visual, que permiten actualizar los sistemas de pensamiento de nuestra época y reflexionar sobre ellos, ponerlos en contexto y conocerlos, para poder ir dando los pasos necesarios en pos de la transformación social.

Quisiéramos finalizar este ensayo, con una cita de Margaret Atwood, quien, ante la pregunta de si su obra era una predicción, respondió: "No, no es una predicción, porque predecir el futuro no es realmente posible: hay demasiadas variables y posibilidades 
imprevistas. Digamos que es una antipredicción: si este futuro se puede describir en detalle, tal vez no suceda ${ }^{3}$...” (2017, s.p).

\section{Figura 1 \\ Blessed Be}

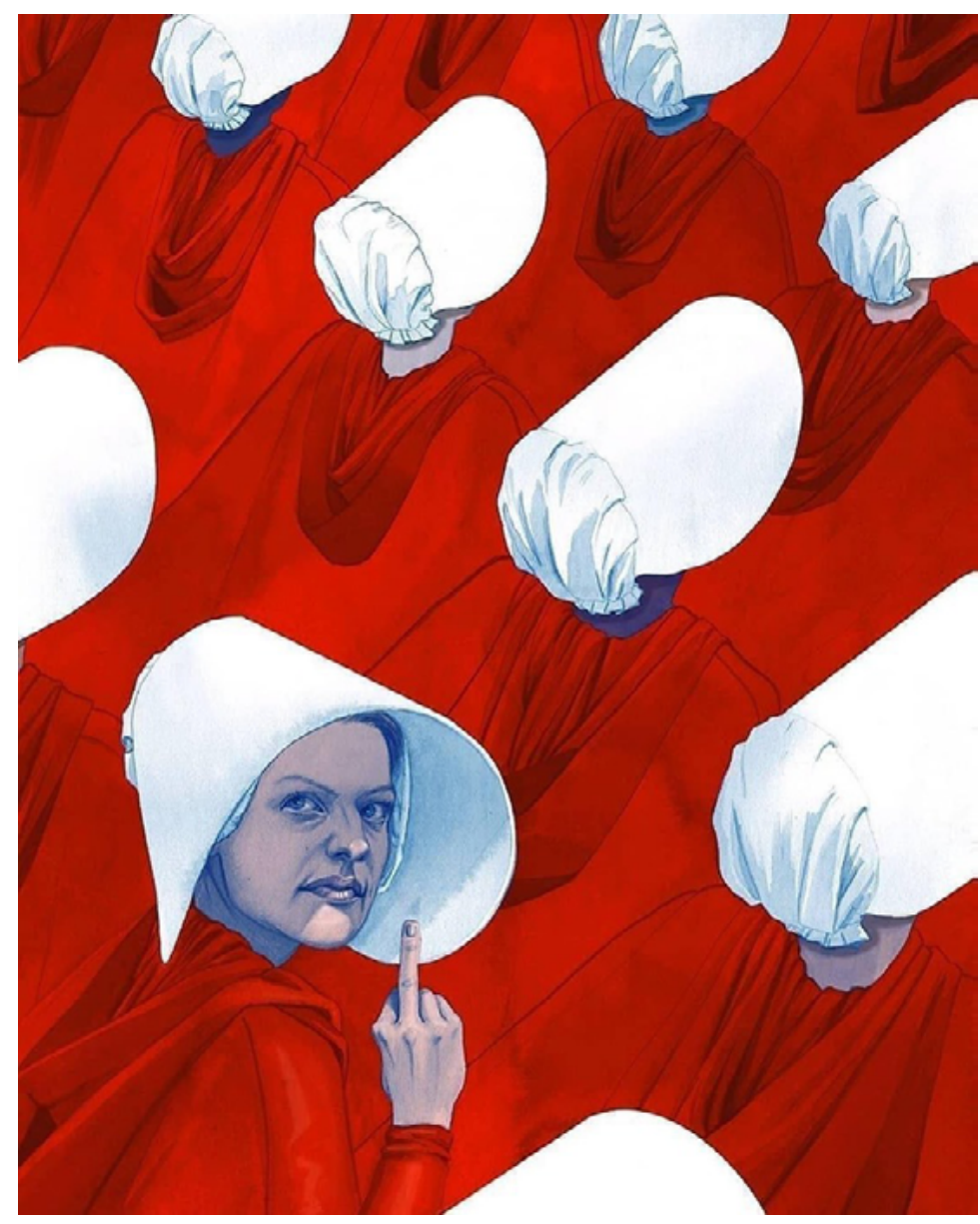

Fuente: obra de la ilustradora Zoë van Dijk (2019)

\section{Referencias bibliográficas}

Agüero, L. (2018). El patriarcado en The Handmaid's Tale. Es De Politólogos. https://bit. ly/2Ch97ck

Atwood, M. (2017). Margaret Atwood on What The Handmaid's Tale' Means in the Age of Trump. The New York Times. https://nyti.ms/3e97RFA

Facio, A., y Fries, L. (2005). Feminismo, género y patriarcado. Academia: revista sobre enseñanza del derecho de Buenos Aires. 3(6), 259-294. https://bit.ly/3gApIqA

3 Traducido del inglés por los autores. 
Fontenla, M. (2008). ¿Qué es el patriarcado? Diccionario de Estudios de Género y Feminismos. Mujeres en Red. El Periódico Feminista. https://bit.ly/2H5tVX2

Granados, A. (2016). En la variedad está el gusto. El feminismo, entre la pluralidad y la reafirmación de los compromisos comunes. Revista CS, (18), 85-106. https://bit.ly/3fbmRnx

Lamas, M. (2000). Diferencias de sexo, género y diferencia sexual. Cuicuilco, 7(18), 1-24. https://bit.ly/2ZOvIW0

Longan, S. (2011). Sobre la definición del arte y otras disquisiciones. Revista Comunicación. 20(1), 75-79. https://bit.ly/2Cjzsqb

Miller, B. (productor). (2017). “The Handmaid's Tale” [serie de televisión]. Estados Unidos, HULU.

Miller, B. (productor). (2018). “The Handmaid's Tale” [serie de televisión]. Estados Unidos, HULU.

Miller, B. (productor). (2019). “The Handmaid's Tale” [serie de televisión]. Estados Unidos, HULU.

Núñez, D. (2017). Feminidades especulativas. Género y política en The Handmaid's Tale. Representaciones. Revista de Estudios sobre Representaciones en Arte, Ciencia y Filosofía. 13(2), 85-105. https://bit.ly/3iIrszK

Pérez, A. (2013). Arte y política. Nuevas experiencias estéticas y producción de subjetividades. Comunicación Y Sociedad, (20), 191-210. https://bit.ly/2O3C1zB

Seoane, A. (2017). Margaret Atwood: “Sin la esclavitud Europa sería inviable”. El Cultural. https://bit.ly/2Dl0sq0 